\title{
Investigating Some Technical Issues on Cohesive Zone Modeling of Fracture
}

\author{
John T. Wang ${ }^{1}$ \\ NASA Langley Research Center, Hampton, VA, 23693
}

\begin{abstract}
This study investigates some technical issues related to the use of cohesive zone models (CZMs) in modeling fracture processes. These issues include: why cohesive laws of different shapes can produce similar fracture predictions; under what conditions CZM predictions have a high degree of agreement with linear elastic fracture mechanics (LEFM) analysis results; when the shape of cohesive laws becomes important in the fracture predictions; and why the opening profile along the cohesive zone length needs to be accurately predicted.

Two cohesive models were used in this study to address these technical issues. They are the linear softening cohesive model and the Dugdale perfectly plastic cohesive model. Each cohesive model constitutes five cohesive laws of different maximum tractions. All cohesive laws have the same cohesive work rate (CWR) which is defined by the area under the traction-separation curve. The effects of the maximum traction on the cohesive zone length and the critical remote applied stress are investigated for both models. For a CZM to predict a fracture load similar to that obtained by an LEFM analysis, the cohesive zone length needs to be much smaller than the crack length, which reflects the small scale yielding condition requirement for LEFM analysis to be valid. For large-scale cohesive zone cases, the predicted critical remote applied stresses depend on the shape of cohesive models used and can significantly deviate from LEFM results. Furthermore, this study also reveals the importance of accurately predicting the cohesive zone profile in determining the critical remote applied load.
\end{abstract}

\section{Nomenclature}

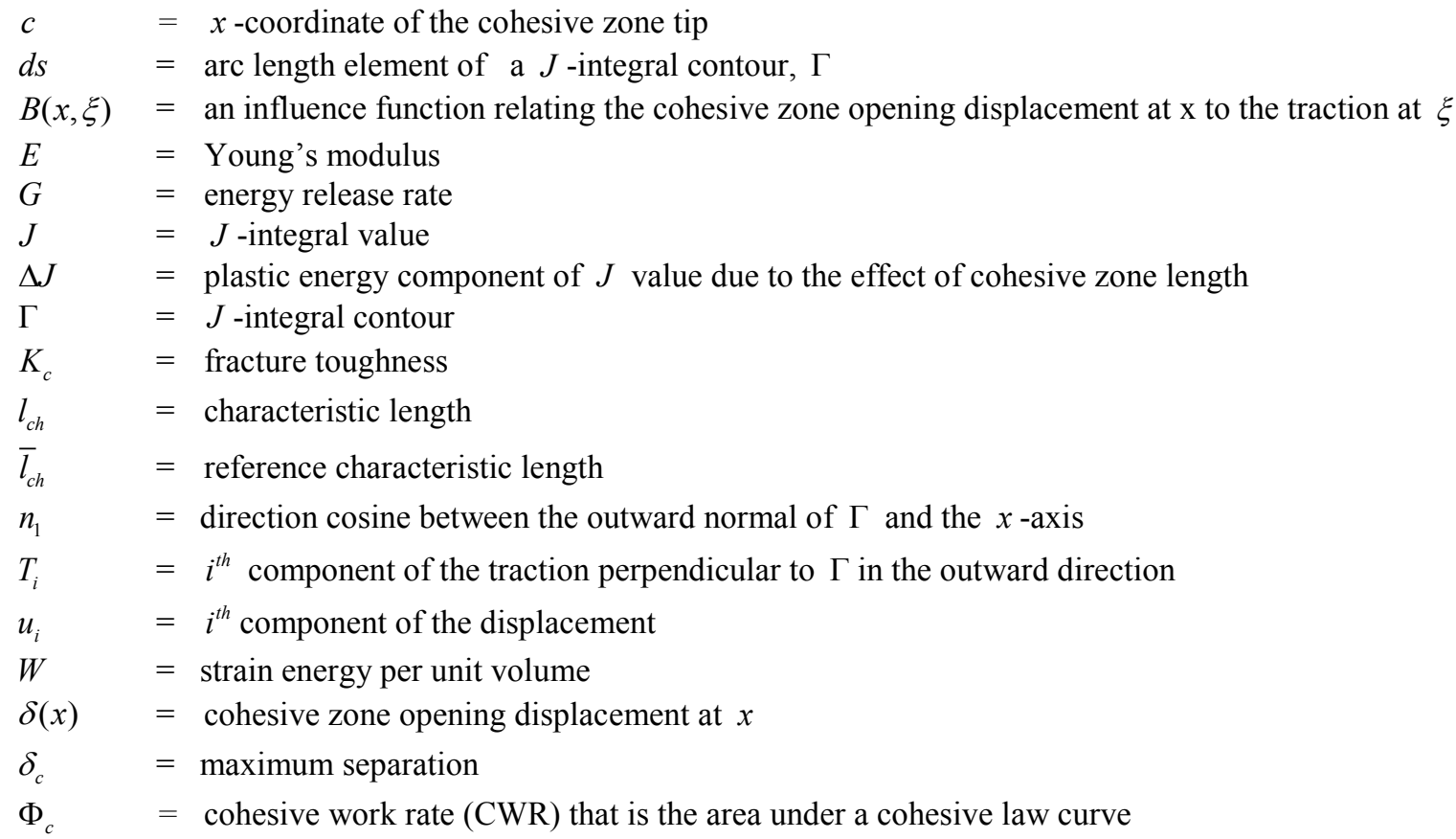

\footnotetext{
${ }^{1}$ Aerospace Engineer, NASA Langley Research Center, Mail Stop 188E, and AIAA Associate Fellow.
} 


$\begin{array}{ll}\rho & =\text { cohesive zone length } \\ \sigma & =\text { cohesive traction } \\ \sigma_{c} & =\text { maximum cohesive traction } \\ \bar{\sigma}_{c} & =\text { reference cohesive traction } \\ \sigma_{\infty} & =\text { remote applied stress } \\ \sigma_{\infty}^{c} & =\text { critical remote applied stress at the initiation of crack growth }\end{array}$

\section{Introduction}

$\mathrm{T}_{\mathrm{c}}^{\mathrm{H}}$ HE origin of cohesive zone models (CZMs) can be traced back to the "Dugdale-Barenblatt model" [1,2]. The cohesive zone is considered to be a fracture process zone ahead of the crack tip as illustrated in Fig. 1 for an infinite plate with a crack. For most cohesive laws, the traction-separation curves, as shown in Fig. 2, used to model the material behavior within the cohesive zone are phenomenological, and hence, may not be directly related to the physical processes in the damage zone that typically are difficult to determine experimentally. Regardless, the CZM approach has been widely accepted as a computationally convenient fracture analysis tool. If the CZM approach is used in a finite element analysis, the crack initiation, growth and direction of the growth can be determined without the involvement of the analyst by properly embedding cohesive elements within the finite element model. Many different cohesive laws with variances in maximum traction, maximum separation, and shape have been proposed, such as the linear softening cohesive law by Camacho and Ortiz [3], the exponential cohesive law by Needleman $[4,5]$ and $\mathrm{Xu}$ and Needleman [6], the trapezoidal cohesive law by Tvergaard and Huchinson [7], and the polynomial cohesive law by Tvergaard [8]. Researchers found these cohesive laws generally produce results that correlated well with experimental data, such as failure load and crack growth.

Although the aforementioned references use cohesive laws with different shapes, they lack justifications for choosing one shape instead of another. Recently, Chandra et al. [9] and Shet et al. [10] found that the shape of the CZM could affect the fracture predictions for a range of material systems. They stated that the shape of the CZM represents the net effect of the failure process and depends on the material systems. For linear elastic materials, the CZM predictions are expected to be comparable to linear elastic fracture mechanics (LEFM) results [11,12, 13]; however, the conditions, under which the cohesive zone modeling approach and the fracture mechanics approach can have a high degree of agreement, need to be more systematically investigated. Here, a high degree of agreement means that the two modeling approaches can predict near the same crack growth initiation load for a cracked structure.

The objectives of this paper are to investigate: why cohesive laws of different shapes can produce similar fracture predictions; under what conditions CZM predictions have a high degree of agreement with LEFM analysis results; when the shape of cohesive laws becomes important in the fracture predictions; and why the opening profile along the cohesive zone length needs to be accurately predicted. In Section II, the linear softening cohesive model (Linear Softening Model) and the Dugdale perfectly plastic cohesive model (Dugdale Model) used in this study are presented. Each model consists of five different cohesive laws (parameterizations of maximum tractions and maximum separations). In Section III, the relationship between the cohesive work rate (CWR), which is the area under the traction-separation curve of a cohesive law, and the J-integral value [11] of the cohesive zone is discussed. In Section IV, integral equations are formulated for using the Linear Softening Model and the Dugdale Model to analyze the fracture of an infinite plate with a crack under a remote tensile load, and the equations are solved with an iterative numerical procedure. This iterative procedure is implemented as a MATLAB ${ }^{\circledR}$ M-file [14] for solving these equations to obtain the length of the cohesive zone ahead of the original crack, the opening displacement profile along the cohesive zone length, and the critical remote applied stress corresponding to the initiation of crack growth. In Section V, the analysis results of Section IV are presented and discussed. The effect of varying the maximum traction of a cohesive law on the predicted cohesive zone length and the critical remote applied stress is investigated. The results from the Linear Softening Model and the Dugdale Model are plotted as a function of the ratio of cohesive zone length to crack length, and these plots are used to address why cohesive laws of different shapes can produce similar fracture predictions and when the shape of the cohesive zone shape matters in determining the critical remote applied stress. The critical remote applied stress is then used in an LEFM formula to determine the energy release rate. If the energy release rate thus obtained is close to the cohesive work rate (CWR), then the failure load predicted by the CZM for a cracked structure will have a high degree of agreement with that obtained by the LEFM approach. In Section VI, concluding remarks are given to summarize the findings of this study. 


\section{Cohesive Zone Models}

The cohesive zone modeling approach is used to study the fracture of an infinite plate with a crack length of $2 a$ subjected to a remote applied tensile stress $\sigma_{\infty}$ as shown in Fig. 1a. The cohesive zone length (CZL)-the process zone length-is given by $\rho$ and the total length $c$ equals $a+\rho$. Note that all results presented in this paper involve Mode I fracture, and the material surrounding the cohesive zone is assumed to be in its linear elastic stage. This may be the simplest case that can be easily solved to provide analytical results needed for investigating the technical issues raised in the previous section. Other boundary value problems (different in geometries or loadings) may yield different quantitative results, but the qualitative trends will be the same. In the cohesive zones (narrow shaded bands shown in Fig. 1) ahead of the material crack tips, the prospective fracture surfaces are assumed to be restrained by a cohesive stress that Dugdale took to be the yield stress of the material [2]. Note the material crack tip shown in Fig. $1 \mathrm{~b}$ is the initial crack tip that is located at $a$ and the cohesive zone tip, where the separation equals zero, is located at $c$. The traction (cohesive stress), $\sigma$, and the separation, $\delta=u_{2}^{+}-u_{2}^{-}$, of the cohesive surfaces are shown in Fig. 1b, where $u_{2}^{+}$and $u_{2}^{-}$are the opening displacements in the y-direction for the upper and lower cohesive surfaces, respectively. In cohesive zone modeling, a traction-separation curve is used to relate the traction, $\sigma$, to the separation, $\delta$, at any location along the $x$-axis for describing the constitutive behavior of the cohesive zone. The linear softening cohesive law shown in Fig. 2a can be expressed as

$$
\begin{array}{cl}
\sigma=\sigma_{c}\left(\delta_{c}-\delta\right) / \delta_{c} & 0 \leq \delta \leq \delta_{c} \\
=0 & \delta>\delta_{c}
\end{array}
$$

where $\sigma_{c}$ is the maximum traction and $\delta_{c}$ is the maximum separation. The $\mathrm{CWR}, \Phi_{c}$, which is the work of separation per unit area, is the area under the linear softening curve given by

$$
\Phi_{c}=\frac{1}{2} \sigma_{c} \delta_{c}
$$

The Dugdale perfectly plastic cohesive law as shown in Fig. $2 \mathrm{~b}$ has the same maximum traction and CWR as the linear softening cohesive law and is given by

$$
\begin{aligned}
\sigma & =\sigma_{c} & & 0 \leq \delta \leq \delta_{c} / 2 \\
& =0 & & \delta>\delta_{c} / 2
\end{aligned}
$$

The sequence of events leading to crack propagation is depicted in Fig. 3 [7]. In Fig. 3a, an initial crack is shown in an unloaded state. Under increased loading, the cohesive zone is fully developed (Fig. 3b) when the maximum cohesive zone opening at the material crack tip (see Fig. 1b), $\delta(a)$, equals the maximum separation, $\delta_{c}$. Further loading will create new crack surfaces, and both the material crack tip and the cohesive zone tip will advance. The length $\Delta a$ in Fig. 3c represents the extension due to crack growth. During the crack growth, the length of the cohesive zone and the opening displacement profile along the length of the cohesive zone are assumed to be unchanged.

The results from the Linear Softening Model and the Dugdale Model are used for investigating some technical issues related to cohesive zone modeling of fracture. These issues include: why cohesive laws of different shapes can produce similar fracture predictions; under what conditions CZM predictions have a high degree of agreement with LEFM analysis results; when the shape of cohesive laws becomes important in the fracture predictions; and why the opening profile along the cohesive zone length needs to be accurately predicted. Five linear softening cohesive laws (Cohesive Laws A to E in solid lines) and five Dugdale perfectly plastic cohesive laws (Cohesive Laws $\mathrm{A}$ to $\mathrm{E}$ in dashed lines) used in this study are shown in Fig. 4. These cohesive laws have different parameterizations of maximum tractions and maximum separations, but all have the same CWR. The maximum tractions for Cohesive Laws $\mathrm{A}$ to $\mathrm{E}$ of the Linear Softening Model are $\bar{\sigma}_{c} / 2, \bar{\sigma}_{c}, \sqrt{2} \bar{\sigma}_{c}, 2 \bar{\sigma}_{c}$, and $4 \bar{\sigma}_{c}$, respectively, and their corresponding maximum separations are $2 \bar{\delta}_{c}, \bar{\delta}_{c}, \bar{\delta}_{c} / \sqrt{2}, \bar{\delta}_{c} / 2$, and $\bar{\sigma}_{c} / 4$, respectively. Note that $\bar{\sigma}_{c}$ is a reference traction and $\bar{\delta}_{c}$ is a reference separation. The maximum tractions for Cohesive Laws A to E of the Dugdale Model are $\bar{\sigma}_{c} / 2, \bar{\sigma}_{c}, \sqrt{2} \bar{\sigma}_{c}, 2 \bar{\sigma}_{c}$, and $4 \bar{\sigma}_{c}$, respectively, and their corresponding maximum separations are $\bar{\delta}_{c}, \bar{\delta}_{c} / 2, \bar{\delta}_{c} /(2 \sqrt{2}), \bar{\delta}_{c} / 4$, and $\bar{\sigma}_{c} / 8$, respectively. These cohesive laws are used for computing the fully developed cohesive zone length and the critical remote applied stress for the infinite plate with a crack as shown in Fig. 1a. 


\section{CWR and J-integral Value}

The J-integral, taken along the boundary of a cohesive zone, the contour $\Gamma$, is expressed as [11],

$$
J=\int_{\Gamma}\left(W n_{1}-T_{i} \frac{\partial u_{i}}{\partial x}\right) d s
$$

where $W$ is the strain energy per unit volume, $n_{1}$ is the direction cosine between the positive side (outward) normal of $\Gamma$ and the $x$-axis, $T_{i}$ is the $i^{\text {th }}$ component of the traction perpendicular to $\Gamma$ in the outward direction, $u_{i}$ is the $i^{\text {th }}$ component of the displacement, $d s$ is an arc length element along $\Gamma$.

For contour $\Gamma$ surrounding a fully developed cohesive zone as shown in Fig. 5, $n_{1}=T_{1}=0$ and the $J$-integral value becomes $[11,15]$

$$
\begin{aligned}
J_{c} & =-\int_{a}^{a+\rho} \sigma\left[\frac{\partial u_{2}^{+}}{\partial x}-\frac{\partial u_{2}^{-}}{\partial x}\right] d x=-\int_{a}^{a+\rho} \sigma \frac{\partial \delta}{\partial x} d x \\
& =\int_{0}^{\delta_{c}} \sigma d \delta=\Phi_{c}
\end{aligned}
$$

where $J_{c}$ is the critical $J$ value immediately before the initiation of crack growth. Note that Eq. 5 is valid with the assumption of a zero stress intensity factor at the cohesive zone tip [1,2,16-18]. Without this assumption, the cohesive zone length cannot be uniquely determined. $J_{c}$ equals the CWR, $\Phi_{c}$. Since the $J$-integral is a path independent integral, the $J$ value obtained from any contour integral around the cohesive zone such as $\Gamma_{1}$ in Fig. 5 must also equal to $J_{c}$.

Cohesive zone models of various shapes, maximum tractions, maximum separations, and CWRs can be used to model the fracture processes of both brittle and ductile materials. If the cohesive zone model is used for brittle materials, it must produce results similar to those obtained from LEFM. The stress field outside of the cohesive zone must be the same as the K-dominant stress field $[12,19]$ in the LEFM model. Similar to the small-scale yielding requirement for an LEFM approach to be valid, the cohesive zone length must be much less than the crack length in order for the CZM approach to produce a fracture prediction comparable to LEFM analysis. The small-scale cohesive zone is required for maintaining the K-dominant stress field [12]. For small-scale cohesive zone cases, $J_{c}$ is approximately equal to the critical energy release rate, $G_{c}$, as defined in LEFM. This is the reason why many cohesive laws assume that the CWR equals the critical energy release rate often used for fracture analyses of brittle materials including polymeric composites [20-23], i.e.

$$
J_{c}=\Phi_{c} \approx G_{c}=\frac{\left(\sigma_{\infty}^{c}\right)^{2} \pi a}{E}
$$

where $\sigma_{\infty}^{c}$ is the critical remote applied stress required for initiating a crack growth, and $a$ is the crack length as defined in Fig. 1a.

Analyses using cohesive laws having the same CWR but different shapes will result in the same $J_{c}$. If $J_{c}$ is the only parameter to determine fracture, then the shape of cohesive laws is not important in the fracture predictions. This is the reason why CZMs with different shapes can generate similar failure predictions for brittle fracture.

If a CZM is used for modeling the fracture of ductile materials, it is necessary to use a cohesive law that can produce a long cohesive zone length to represent the behavior of large scale yielding in these materials. The $J_{c}$, computed along contour $\Gamma_{1}$ in Fig. 5 at a critical remote applied stress $\left(\sigma_{\infty}\right)$, may include both elastic and plastic components similar to the relationship shown in Eq. 3.29 of Reference 24. For such a cohesive zone model, $J_{c}$ may be expressed as

$$
\begin{aligned}
J_{c} & =\frac{K_{c}^{2}}{E}+\Delta J\left(\frac{\rho}{a}\right)=\frac{\left(\sigma_{\infty}^{c}\right)^{2} \pi a}{E}+\Delta J\left(\frac{\rho}{a}\right) \\
& =G\left(\sigma_{\infty}^{c}\right)+\Delta J\left(\frac{\rho}{a}\right)
\end{aligned}
$$


where $K_{c}$ is a fracture toughness term, $G\left(\sigma_{\infty}^{c}\right)=\frac{\left(\sigma_{\infty}^{c}\right)^{2} \pi a}{E}$ is the elastic component, and $\Delta J$ is the plastic component which is assumed to be a function of the scale of the cohesive zone. Here, $\sigma_{\infty}^{c}$ is the critical remote applied stress. Note that unlike LEFM, there is no simple relationship between $J_{c}$, the critical remote applied stress, the cohesive zone length and the crack length. For a fully developed cohesive zone, $J_{c}$ equals $\Phi_{c}$, and Eq. 7 can be rewritten as

$$
\begin{aligned}
J_{c} & =\Phi_{c}=G\left(\sigma_{\infty}^{c}\right)+\Delta J\left(\frac{\rho}{a}\right) \\
& =\frac{\left(\sigma_{\infty}^{c}\right)^{2} \pi a}{E}+\Delta J\left(\frac{\rho}{a}\right)
\end{aligned}
$$

For clarity, Eq. (8) can be expressed as

$$
\frac{G\left(\sigma_{\infty}\right)}{\Phi_{c}}=1-\frac{\Delta J\left(\frac{\rho}{a}\right)}{\Phi_{c}}
$$

The second term of this equation includes the effect of the scale of cohesive zone, $\rho / a$. The validity of Eq. (9) will become clear after examining the CZM results in Section V. For nearly brittle fracture, $\rho / a<<1$ and $G\left(\sigma_{\infty}\right) \approx \Phi_{c}$, thus $\Delta J\left(\frac{\rho}{a}\right) \rightarrow 0$. The closer $\rho / a$ is to zero, the closer CZM predictions approach those of LEFM.

\section{Analytical Procedures}

The cohesive zone is treated as an extended part of the crack with its fully developed length determined by the requirement that the stress intensity factor vanishes at the tip of the cohesive zone [1,2,16-18]. Additional information on the requirement of a zero stress intensity factor at the cohesive zone tip can be found in Reference 1. For the infinite plate with a crack shown in Fig. 1a, the following integral equation [18] can be used to determine the remote applied stress, $\sigma_{\infty}$ for a given cohesive zone length, $c-a$.

$$
\sigma_{\infty} \sqrt{\pi c}-\frac{2}{\sqrt{\pi c}} \int_{a}^{c} \frac{\sigma(\xi) d \xi}{\sqrt{1-\xi^{2} / c^{2}}}=0
$$

where $c$ is the $x$-coordinate of the cohesive zone tip and $\sigma(\xi)$ is the traction at location $\xi$ as shown in Fig. $1 b$. Note that the cohesive zone tip location $c$ is an unknown, so for any given cohesive zone length $c-a$, there is a corresponding value of $\sigma_{\infty}$ that can satisfy Eq. (10). The $\sigma_{\infty}$ being sought is the value that can induce an opening displacement $\delta(a)=\delta_{c}$ at the material crack tip $a$ (see Figs. $1 \mathrm{~b}$ and $3 \mathrm{~b}$ ).

The cohesive zone opening displacement $\delta$ at location $x$ is [18]

$$
\delta(x)=\frac{4 c \sigma_{\infty}}{E} \sqrt{1-\frac{x^{2}}{c^{2}}}-\frac{4}{\pi E} \int_{a}^{c} B(x, \xi) \sigma(\xi) d \xi
$$

where $E$ is the Young's modulus, and $B(x, \xi)$ is an influence function given by [25]

$$
B(x, \xi)=\ln \left|\frac{\sqrt{1-x^{2} / c^{2}}+\sqrt{1-\xi^{2} / c^{2}}}{\sqrt{1-x^{2} / c^{2}}-\sqrt{1-\xi^{2} / c^{2}}}\right|
$$

For the Linear Softening Model shown in Fig. 2a, Eq. (11) can be expressed as

$$
\delta(x)=\frac{4 c \sigma_{\infty}}{E} \sqrt{1-\frac{x^{2}}{c^{2}}}-\frac{4}{\pi E} \int_{a}^{c} B(x, \xi) \frac{\sigma_{c}\left(\delta_{c}-\delta(\xi)\right)}{\delta_{c}} d \xi
$$

and Eq. (10) can be expressed as 


$$
\sigma_{\infty}=\frac{2 \sigma_{c}}{\pi c}\left(-\int_{a}^{c} \frac{\delta(\xi) / \delta_{c}}{\sqrt{1-\xi^{2} / c^{2}}} d \xi+\int_{a}^{c} \frac{d \xi}{\sqrt{1-\xi^{2} / c^{2}}}\right)
$$

Substituting $\sigma_{\infty}$ into Eq. (13), an integral equation is obtained for determining the cohesive zone length, $\rho=c-a$, and cohesive zone opening displacements, $\delta(x)$

$$
\begin{aligned}
& \frac{\pi E \delta^{*}(x) \Phi_{c}}{2 c \sigma_{c}^{2}}-\frac{1}{c} \int_{a}^{c} B(x, \xi) \delta^{*}(\xi) d \xi+\frac{2}{c} \sqrt{1-\frac{x^{2}}{c^{2}}} \int_{a}^{c} \frac{\delta^{*}(\xi)}{\sqrt{1-\xi^{2} / c^{2}}} d \xi \\
& =\frac{2}{c} \sqrt{1-\frac{x^{2}}{c^{2}}} \int_{a}^{c} \frac{d \xi}{\sqrt{1-\xi^{2} / c^{2}}}-\frac{1}{c} \int_{a}^{c} B(x, \xi) d \xi
\end{aligned}
$$

where $\delta^{*}(x)=\delta(x) / \delta_{c}$. Eq. (15) can be written as

$$
\delta^{*}(x)=\frac{2}{\pi}(c / a)\left(a / l_{c h}\right)\left[\frac{1}{c} \int_{a}^{c} B(x, \xi) \delta^{*}(\xi) d \xi-\frac{2}{c} \sqrt{1-\frac{x^{2}}{c^{2}}} \int_{a}^{c} \frac{\delta^{*}(\xi)}{\sqrt{1-\xi^{2} / c^{2}}} d \xi+\frac{2}{c} \sqrt{1-\frac{x^{2}}{c^{2}}} \int_{a} \frac{d \xi}{\sqrt{1-\xi^{2} / c^{2}}}-\frac{1}{c} \int_{a}^{c} B(x, \xi) d \xi\right]
$$

where $l_{c h}$ is a characteristic length [26] defined as,

$$
l_{c h}=\frac{E \Phi_{c}}{\sigma_{c}^{2}}
$$

Note that the characteristic length $l_{c h}$ has been used as an estimate of the cohesive zone length for determining the finite element mesh size [22,27,28] for failure predictions. For the Dugdale Model shown in Fig. 2b, much simpler equations similar to Eqs. (14) to (16) can be obtained due to a constant traction along the cohesive zone length.

Equation 16 can be solved with an iterative procedure [18] outlined in Fig. 6 to determine the cohesive zone length, $\rho=c-a$, for a fully developed cohesive zone and the cohesive zone opening displacements $\delta(x)$. Note that Eq. (16) only needs two dimensionless input parameters, $c / a$ and $a / l_{c h}$. To start the iterative procedure, an initial cohesive zone length is selected to provide an estimate of the integral interval, $[a, c]$, for the integrals in Eq. (16) to determine the cohesive zone opening displacements along the given cohesive zone length. If the predicted opening displacement at the material crack tip $\delta(a)$ is not equal to the maximum separation $\delta_{c}$, an updated cohesive zone length is obtained by adding a small increment $\Delta \rho$, and new cohesive zone opening displacements along the given cohesive zone length are determined by repeating the numerical solution of Eq. 16. This iterative procedure is continued until $\delta(a)$ equals $\delta_{c}$, at this point the cohesive zone is fully developed as shown in Fig. $3 \mathrm{~b}$. The final value of $c-a$ is the predicted cohesive zone length and the associated remote applied stress $\sigma_{\infty}$ is designated as the critical remote applied stress $\sigma_{\infty}^{c}$. The critical remote applied stress $\sigma_{\infty}^{c}$ can be obtained with Eq. (14), by using the final set of cohesive zone opening displacements. The critical remote applied stress is used to compute the energy release rate using the LEFM formula given in Eq. 6. If the ratio of the computed energy release rate $G\left(\sigma_{\infty}^{c}\right)$ to the CWR, $\Phi_{c}$, is close to unity, then the prediction using the CZM approaches LEFM prediction of fracture.

\section{Analytical Results and Discussions}

Analytical results for cohesive laws of the Linear Softening Model and the Dugdale Model as shown in Fig. 4 are presented in this section. All cohesive laws have the same CWR, but different maximum tractions. All the results are computed at the initiation of crack growth when the cohesive zone is fully developed. The effects of maximum traction on the cohesive zone length, the remote applied stress, and the degree of correspondence of the CZM approach and LEFM analyses are presented. The effect of maximum traction on the cohesive zone length can be found in Fig. 7, which shows that larger maximum tractions result in shorter cohesive zone lengths. The cohesive zone lengths are obtained for two different crack lengths, one has $a / \bar{l}_{c h}=1$ and the other one has $a / \bar{l}_{c h}=10$, where $\bar{l}_{c h}$ is a reference characteristic length defined as 


$$
\bar{l}_{c h}=\frac{E \Phi_{c}}{\bar{\sigma}_{c}^{2}}
$$

Two solid line curves in the figure represent the Linear Softening Model results. The two solid line curves in Fig. 7 merge into one for maximum tractions greater than $2 \bar{\sigma}_{c}$. This indicates that the cohesive zone length does not depend on the crack length for a cohesive law with a large maximum traction. However, the cohesive zone lengths depend on crack length for models with a maximum traction less than $2 \bar{\sigma}_{c}$. The cohesive zone length for the short crack $a / \bar{l}_{c h}=1$ is longer than that of the long crack $a / \bar{l}_{c h}=10$. The two dashed line curves in Fig. 7 represent Dugdale Model results for crack length of $a / \bar{l}_{c h}=1$ and $a / \bar{l}_{c h}=10$. In general, the cohesive zone length obtained with a Dugdale Model is less than the length obtained with its corresponding Linear Softening Model. These curves also show that the cohesive zone length does not depend on crack length for a cohesive law with a large maximum traction. A few following figures will use the data presented in this figure.

Cohesive zone lengths are predicted for all cohesive laws in Fig. 4 for different crack lengths ranging from $a / \bar{l}_{c h}=0.25$ to $a / \bar{l}_{c h}=16$ and plotted in Fig. 8. For clarity, only results of Cohesive Laws A, B, and D are plotted in Fig. 8. Cohesive Law A represents the most ductile material behavior, Cohesive Law D represents the most brittle material behavior, and Cohesive Law B represents an intermediate material behavior. Cohesive Laws A, B, and D have maximum tractions of $\bar{\sigma}_{c} / 2, \bar{\sigma}_{c}$, and $2 \bar{\sigma}_{c}$, respectively. It is apparent that each cohesive-zone-length curve for either the Linear Softening Model (solid line) or the Dugdale Model (dashed line) will reach a constant value as the crack length increases. For the cohesive law with the largest maximum traction, Cohesive Law D, the cohesive zone length reaches a constant value at a crack length around $a=2 \bar{l}_{c h}$. For the second largest maximum traction, Cohesive Law B, the cohesive zone length reaches a constant value at a crack length around $a=5 \bar{l}_{c h}$. The cohesive zone length almost reaches a constant value at $a=16 \bar{l}_{c h}$ for the smallest maximum traction, Cohesive Law A. The cohesive zone length curves (dashed lines) of the Dugdale Model are in general lower than the cohesive zone length curves (solid lines) of the Linear Softening Model if the crack length is not very short.

The cohesive zone opening displacements along the cohesive zone length for cohesive laws with different maximum tractions are plotted in Fig. 9. For both the Linear Softening Model and the Dugdale Model, each model has three cohesive zone opening displacement curves plotted in the figure for three cohesive laws with maximum tractions of $\bar{\sigma}_{c} / 2, \bar{\sigma}_{c}$, and $2 \bar{\sigma}_{c}$, respectively. These cohesive zone opening displacement curves along the cohesive zone length are not linear. The shapes of the cohesive zone opening profiles from both models are cusps. Barenblatt [1] found that the cusp shape is the only cohesive zone opening profile that can have a finite stress (a zero stress intensity factor) at the cohesive zone tip and the top and bottom cohesive surfaces smoothly closed. Barenblatt stated that, for the cusp shape opening profile, the energy released by a small extension at the cohesive zone tip must be zero. This statement was used to prove that the stress intensity factor needs to vanish at the cohesive zone tip. Figure 9 also shows that smaller maximum tractions, representing ductile materials, result in a longer cohesive zone length. Note that the small difference in cohesive zone lengths for the Cohesive Law A of the Linear Softening Model and the Dugdale Model reveals that the cohesive zone length is not proportional to the maximum separation of the cohesive law used (see Cohesive Law A in Fig. 4 for both models). For a crack length shorter than $1 \bar{l}_{c h}$, the cohesive zone length of the Dugdale Model using Cohesive Law A is longer than the cohesive zone length of the Linear Softening Model as shown in Fig. 8. These opening displacements are used in Eq. (14) to compute the critical remote applied stresses.

The computed remote applied stresses are normalized with the reference maximum traction, $\bar{\sigma}_{c}$, and the normalized remote applied stresses are plotted as a function of the maximum traction of the CZM models in Fig. 10. Again, the solid lines are the Linear Softening Model results, and the dashed lines are the Dugdale Model results. There are two curves for each model shown in Fig. 10 representing two different crack lengths. The bottom two curves for the longer crack length of $a / \bar{l}_{c h}=10$ show that the remote applied stresses for both models reach the same constant value for the maximum tractions greater than $\bar{\sigma}_{c}$. The top two curves for the shorter crack length of $a=1 \bar{l}_{c h}$ show that for the remote applied stresses to reach a constant value, the maximum traction needs to be greater than $4 \bar{\sigma}_{c}$. The curve of the Linear Softening Model is lower that the Dugdale Model curve, but they are converging for cohesive laws with high maximum tractions. This indicates that the critical remote applied stress is independent of the shape of cohesive laws if their maximum tractions are high, representing brittle material behavior. The 
normalized remote applied stresses are plotted as a function of the normalized cohesive zone length, $\rho / a$, in Fig. 11 for two different crack lengths, $a=\bar{l}_{c h}$ and $a / \bar{l}_{c h}=10$. For $a=\bar{l}_{c h}$, the dashed curve for the Dugdale Model is higher than the solid curve for the Linear Softening Model, but the two curves are converged for small scale cohesive zone lengths, $\rho / a<<1$. This indicates that both models can produce a similar critical remote applied stress, independent of the shape of the cohesive law used, if the small-scale cohesive zone is valid. For $a=\bar{l}_{c h}$, both models predict that the normalized remote applied stress reduces as the cohesive zone length increases. For $a=10 \bar{l}_{c h}$, both models predict near the same normalized remote applied stress results which decrease as the cohesive zone length increases. Curves for different crack lengths converge to their corresponding LEFM solutions for $\rho / a \rightarrow 0$ as shown in Fig. 11. This shows that when the scale of the cohesive zone is small, CZM prediction is similar to LEFM results, and the shape of cohesive law is not important.

The computed remote applied stresses are then used in Eq. (6) to compute LEFM energy release rates $G\left(\sigma_{\infty}^{c}\right)$. The ratio of $G\left(\sigma_{\infty}^{c}\right)$ to $\Phi_{c}$ is defined as the normalized LEFM energy release rate. If the normalized LEFM energy release rate approaches unity, then the cohesive zone model is considered to agree with an LEFM analysis (see Eq. 9). Hence, both analysis approaches would predict similar critical remote applied stress. The normalized LEFM energy release rate as a function of maximum traction is plotted in Fig. 12. The figure shows that for a long crack length, $a / \bar{l}_{c h}=10$, the normalized LEFM energy release rate approaches unity for maximum tractions greater than $2 \bar{\sigma}_{c}$ while for a short crack length, $a=\bar{l}_{c h}$, the normalized LEFM energy release rate reaches unity at a much slower rate, and a cohesive law with a maximum traction greater than $4 \bar{\sigma}_{c}$ needs to be used. Fig. 12 shows that the Dugdale Model results (dashed lines) approach unity faster than the Linear Softening Model results (solid lines) for both crack lengths. This is expected because for the same maximum traction, the cohesive zone length for the Dugdale Model is shorter than the Linear Softening Model as shown in Fig. 7. The Dugdale Model represents more brittle material behavior. The separation of the solid curve and the dashed curve for the same crack length indicates that the shape of the cohesive laws can affect the failure predictions, especially using cohesive laws with a low maximum traction.

The normalized LEFM energy release rate requires high maximum tractions to reach unity for the shorter crack length, $a=\bar{l}_{c h}$. Rice [11] and Hutchinson [12] have stated that a CZM and LEFM can have a high degree of agreement only for materials that have small-scale cohesive zones, brittle materials. It is expected that a large-scale cohesive zone length, similar to a large-scale yielding zone, can significantly alter the original $\mathrm{K}$ dominant stress field around the original material crack tip [12,19]. Hence, the result from a CZM, producing a large cohesive zone length relative to the crack length, cannot agree with an LEFM analysis. Similar to the equation established in Reference 24 for computing the $J$ value for an elastic-plastic material, the relationship between the normalized LEFM energy release rate and the scale of the cohesive zone length is proposed in Eq. 9. The normalized LEFM energy release rate as a function of the ratio of cohesive zone length to crack length, $\rho / a$, is plotted in Fig. 13 for both crack lengths for validating Eq. 9. This plot reveals that the two curves shown in Fig. 12 for the Linear Softening Model can be collapsed to become a single curve as expected by Eq. 9. Similarly, the other curves in the same figure for the Dugdale Model can also be collapsed into a single curve. This indicates that the ratio of the cohesive zone length to the crack length is an important parameter for fracture predictions using a CZM. The ratio of cohesive zone length to crack length, $\rho / a$, must be close to zero for the CZM to be close to an LEFM analysis. Physically, this means that regions ahead of the crack tip that exhibit mechanisms other than brittle fracture (plasticity, bridging fibers, etc) must be very small relative to the crack length for the CZM approach to be close to LEFM.

Figure 13 shows that the normalized LEFM energy release rate curves from the Linear Softening Model and the Dugdale Model separate for large-scale cohesive zone lengths. This indicates that the fracture predictions depend on the shape of the cohesive laws used for large-scale cohesive zone length cases. Since both the Linear Softening Model and the Dugdale Model used have the same area, the CWR is thus not the only parameter determining failure for large-scale cohesive zone length cases. It is apparent that the shape of the cohesive law, the maximum traction or the maximum separation can affect the failure predictions. These observations imply that for large-scale cohesive zone length cases, the cohesive laws need to be determined experimentally. Methods and procedures for determining the bridging laws based on the J-integral approach can be used to obtain the cohesive laws $[29,30]$. 
For illustrating why the cohesive zone opening profile needs to be accurately predicted for determining the critical remote applied stress, the sensitivity of the computed critical remote applied stress relative to the variation of the opening profile is studied. In this study, the cusp shape cohesive-zone-opening profiles for the Linear Softening model as shown in Fig. 9 is replaced with a triangular shape cohesive-zone-opening profile as shown in the inset of Fig. 14. Note the separations at the material crack tip and the cohesive zone length are kept the same. Using the modified cohesive-zone-opening displacements from the triangular shape and Eq. (14), new remote applied stresses are computed. The changes of remote applied stresses relative to the original remote applied stresses are plotted in Fig. 14. The two curves, shown in Fig. 14, represent two different crack lengths, $a=\bar{l}_{c h}$ and $a / \bar{l}_{c h}=10$, respectively. The changes of remote applied stresses are near constant about $9 \%$ for CZM models with maximum traction greater than $2 \bar{\sigma}_{c}$ for both crack lengths. The implication of this study may explain why when using CZM in finite element analysis [28], the failure load predicted is sensitive to mesh size. A finite element model with a fine mesh along the cohesive zone length is needed to get a converged solution. The mesh sensitivity issue is due in part to the inaccurate prediction of the opening profile along the cohesive zone length when using a coarse mesh.

\section{Concluding Remarks}

A few technical issues related to the cohesive zone modeling for fracture predictions of cracked structures were investigated in this paper. These issues include why cohesive laws of different shapes can produce similar fracture predictions; under what conditions CZM predictions have a high degree of agreement with LEFM analysis results; when the shape of cohesive laws becomes important in the fracture predictions; and why the opening profile along the cohesive zone length needs to be accurately predicted. To investigate these technical issues, two cohesive models were used to analyze an infinite plate with a center crack. These models are the Linear Softening Model and the Dugdale Model. Each cohesive model consists of five cohesive laws with different maximum tractions and maximum separations. All cohesive laws have the same CWR, area under the traction-separation curve. MATLAB ${ }^{\circledR}$ M-files were implemented for numerically solving the CZM integral equations of the two models with an iterative procedure.

The effects of maximum traction on the scale of the cohesive zone length and the predicted critical remote applied stress were presented for both cohesive models. This study found that using a cohesive law with high maximum traction results in short cohesive zone lengths. The conditions required for a CZM to predict the critical remote applied stress of a cracked structure compatible to that obtained by a linear elastic fracture mechanics (LEFM) analysis were investigated in this paper. Similar to the small-scale yielding condition for an LEFM analysis to be valid, the cohesive zone length also needs to be about an order of magnitude less than the crack length for the CZM result to be close to the LEFM prediction. For small-scale cohesive zone lengths, the shape has negligible effect on the critical remote applied stress. These observations may be used to explain why many cohesive laws of different shape can produce similar fracture predictions. For large-scale cohesive zone lengths, the predicted critical remote applied stress depends on the shape of the cohesive models and can significantly deviate from the LEFM result due to plasticity like behavior. Thus, the predicted fracture load depends on the shape of the cohesive laws as well as their maximum tractions and separations. Therefore, for large-scale cohesive zone length cases, the cohesive laws need to be determined experimentally. Furthermore, the importance of accurately predicting the cohesive zone opening profile was also investigated. Results show that a small variation in the profile can significantly affect the critical remote applied stress. This suggests that finite element analysis results using a CZM are sensitive to mesh size since a coarse mesh model cannot accurately represent the opening profile along the cohesive zone length.

\section{Acknowledgments}

The author would like to acknowledge the helpful discussions with Professors C. T. Sun of Purdue University and Z. H. Jin of The University of Maine.

\section{References}

${ }^{1}$ Barenblatt, G.I., "The mathematical theory of equilibrium cracks in brittle fracture," Advances in Applied Mechanics, Vol. 7. Academic Press, New York, 1962, pp. 55-129.

${ }^{2}$ Dugdale, D.S., "Yielding of steel sheets containing slits," Journal of the Mechanics and Physics of Solids, Vol. 8, 1960, pp. $100-104$.

${ }^{3}$ Camacho, G.T., and Ortiz, M., "Computational modelling of impact damage in brittle materials," International Journal of Solids and Structures, Vol. 33, 1996, pp. 2899-2938.

${ }^{4}$ Needleman, A., "A continuum model for void nucleation by inclusion debonding," ASME Journal of Applied Mechanics, Vol. 54, 1987, pp. 525-531. 
${ }^{5}$ Needleman, A., “'An analysis of decohesion along an imperfect interface,’ International Journal of Fracture, Vol. 42, 1990, pp. $21-40$.

${ }^{6}$ Xu, X.P., and Needleman, A., "Void nucleation by inclusions debonding in a crystal matrix," Modelling and Simulation in Materials Science and Engineering, Vol. 1, 1993, pp. 111-132.

${ }^{7}$ Tvergaard, V., and Hutchinson, J.W., "The relation between crack growth resistance and fracture process parameters in elastic-plastic solids," Journal of the Mechanics and Physics of Solids, Vol. 40, 1992, pp. 1377-1397.

${ }^{8}$ Tvergaard, V., "Effect of fibre debonding in a whisker-reinforced metal," Materials Science and Engineering A, Vol. 125, 1990, pp. 203-213.

${ }^{9}$ Chandra N., Li. H., Shet, C., and Ghonem, H., "Some issues in the application of cohesive zone models for metal ceramic interfaces," International Journal of Solids and Structures, Vol. 39, 2002, pp. 2827-2855.

${ }^{10}$ Shet, C., and Chandra, N., "Effect of the shape of traction-displacement cohesive zone curves on the fracture response," Mechanics of Advanced Materials and Structures, Vol. 11, 2004, pp. 249-275.

${ }^{11}$ Rice, J.R., "A path independent integral and approximate analysis of strain concentration by notches and cracks," $A S M E$ Journal of Applied Mechanics, Vol. 35, 1968, pp. 379-386.

${ }^{12}$ Hutchinson, J.W., A course on nonlinear fracture mechanics, Department of Solid Mechanics, The Technical University of Denmark, Lyngby, Denmark, 1979, pp. 12-13.

${ }^{13}$ Bao, G., and Suo, Z., "Remarks on crack-bridging concepts," Applied Mechanics Review, Vol. 45, No. 8, August, 1992, pp. 355-366.

${ }^{14} \mathrm{MATLAB}^{\circledR} \quad$ (a high-performance technical computing environment), product of The MathWorks Inc., http://www.mathworks.com.

${ }^{15}$ Kanninen, M.F., and Popelar, C.H., Advanced Fracture Mechanics, Oxford University Press, New York, 1985.

${ }^{16}$ Cox, B.N., and Marshall, D.B., "Concepts for bridged cracks in fracture and fatigue," Acta Metallurgica et Materialia, Vol. 42, No. 2, 1994, pp. 341-363.

${ }^{17}$ Planas, J., and Elices, M., “Asymptotic analysis of cohesive crack: 1. Theoretical background," International Journal of Fracture, Vol. 55, 1992, pp. 153-177.

${ }^{18}$ Jin, Z.H., and Sun, C.T., "Cohesive fracture model based on necking," International Journal of Fracture, Vol. 134, 2005, pp. 91-108.

${ }^{19}$ Broek, D., Elementary Engineering Fracture Mechanics, $4^{\text {th }}$ revised edition, Martinus Nijhoff Publishers, Dordrecht, The Netherlands, 1986, pp. 247-248.

${ }^{20}$ Davila, C.G., Camanho, P.P., and de Moura, M.F., "Mixed-mode decohesion elements for analyses of progressive delamination," In: $42^{\text {nd }}$ AIAA/ASME/ASCE/AHS/ASC Structures, Structural dynamics \& Materials Conference, AIAA-20011486, Seattle, WA, April16-19, 2001.

${ }^{21}$ Camanho P.P., Davila C.G., and de Moura M.F., "Numerical simulation of mixed-mode progressive crack in composite materials," Journal of Composite Materials, Vol. 37, 2003, pp. 1415-38.

${ }^{22}$ Yang, Q., and Cox, B., "Cohesive models for damage evolution in laminated composites," International Journal of Fracture, Vol. 133, 2005, pp. 107-137.

${ }^{23}$ Xie, D., and Waas, A.M., "Discrete cohesive zone model for mixed-mode fracture using finite element method," Engineering Fracture Mechanics, Vol. 73, 2006, pp. 1783-1796.

${ }^{24}$ Anderson, T.L., Fracture Mechanics-Fundamentals and Applications, Third Edition, Taylor \& Francis, Boca Raton, FL, 2005, p. 117

${ }^{25}$ Tada, H., Paris, P.C., and Irwin, G.R., The Stress Analysis of Cracks Handbook. ASME Press, New York, New York, 2000, p. 141.

${ }^{26}$ Hillerborg, A., Modeer, M., and Petersson, P.E., "Analysis of crack formation and crack growth in concrete by means of fracture mechanics and finite elements," Cement and Concrete Research, Vol. 6, 1976, pp. 773-782.

${ }^{27}$ Moës, N., and Belytschko, T., "Extended finite element method for cohesive crack growth," Engineering Fracture Mechanics, Vol. 69, 2002, pp. 813-833.

${ }^{28}$ Turon, A., Davila, C.G., Camanho, P.P., and Costa, J., "An engineering solution for mesh size effects in the simulation of delamination using cohesive zone models," Engineering Fractures Mechanics, Vol. 74, 2007, pp. 1665-1668.

${ }^{29}$ Bao, Z., and Zok, F., "On the strength of ductile particle reinforced brittle matrix composites," Acta Metallurgica Materiali, Vol. 41, No. 12, 1993, pp. 3515-3524.

${ }^{30}$ Sorensen, B.F., and Jacobsen, T.K., "Large-scale bridging in composites: R-curves and bridging laws," Composites Part A, Vol. 29A, 1998, pp. 1443-1451. 


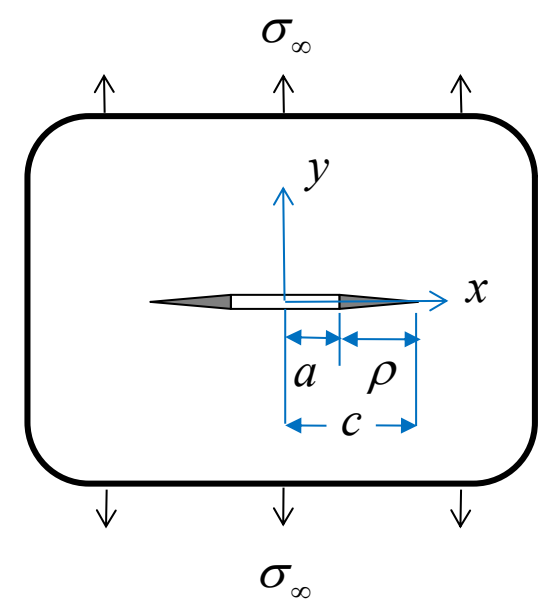

a) Cohesive zone at crack tips

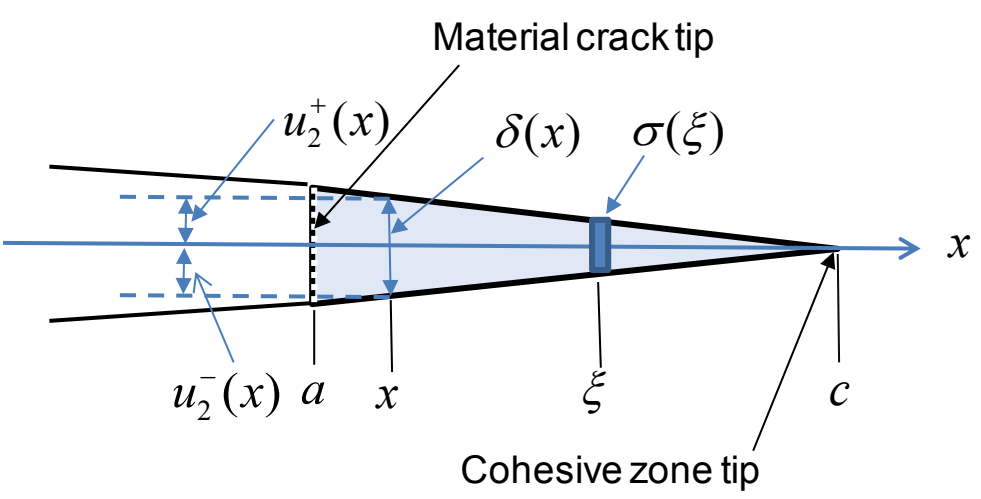

b) Cohesive traction $\sigma$ and cohesive zone opening displacement $\delta$

Fig. 1 Fracture analysis of a cracked infinite plate using a cohesive zone model.

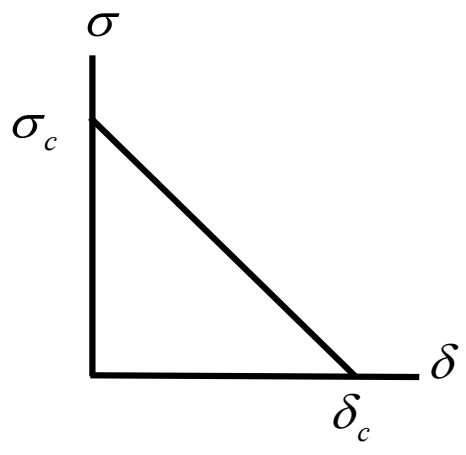

a) Linear softening cohesive law

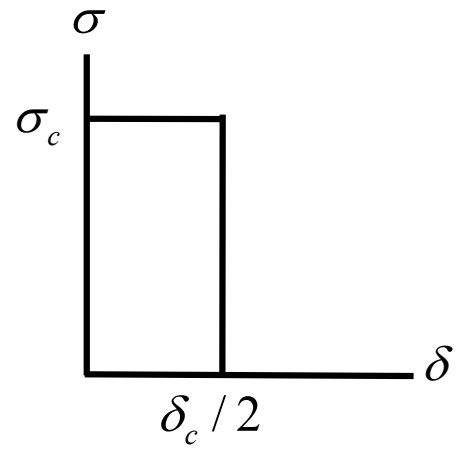

b) Dugdale perfectly-plastic cohesive law

Fig. 2 Linear softening and Dugdale cohesive models. 


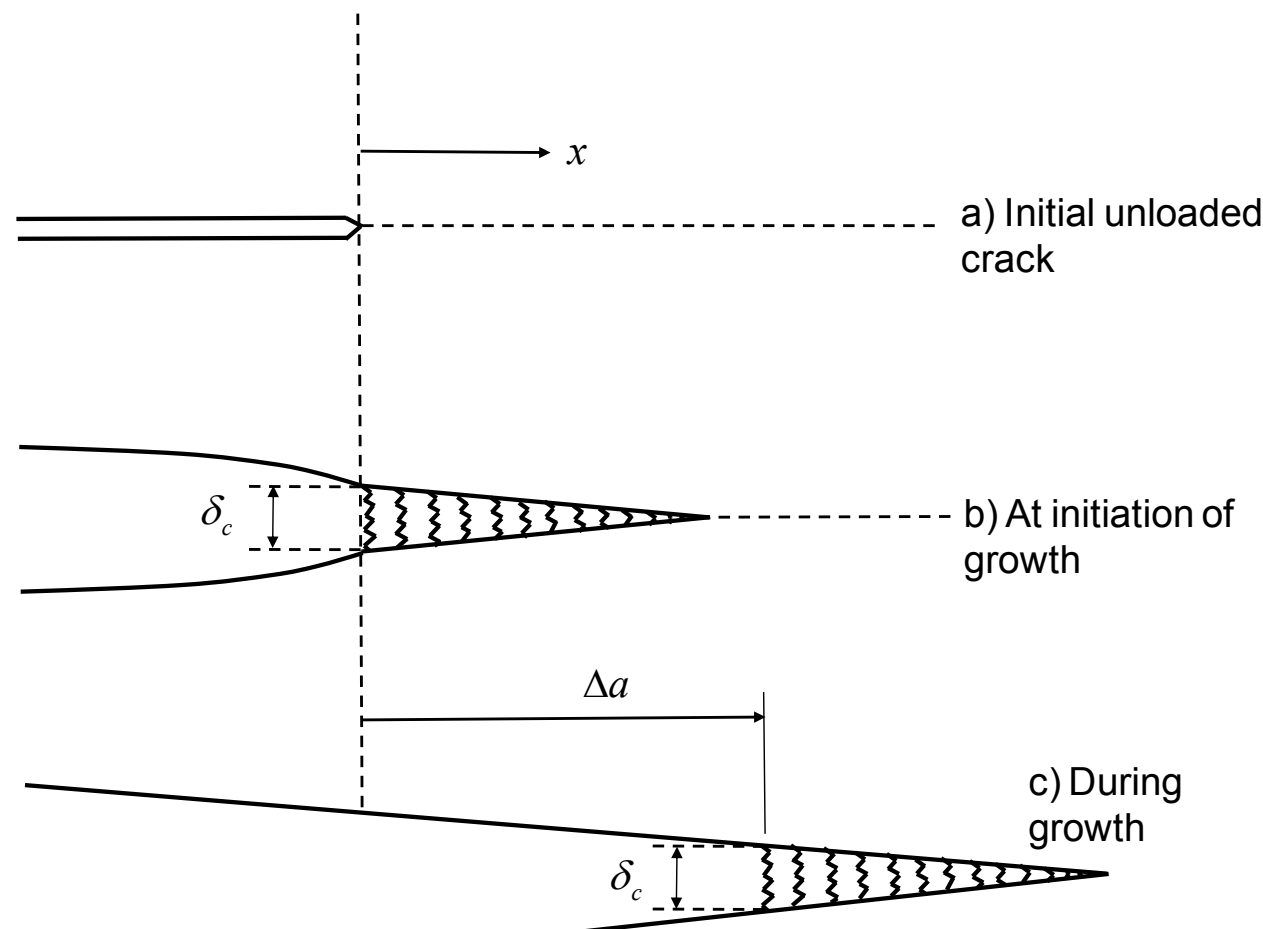

Fig. 3 Cohesive zone fully developed at crack growth initiation and unchanged during growth [7].
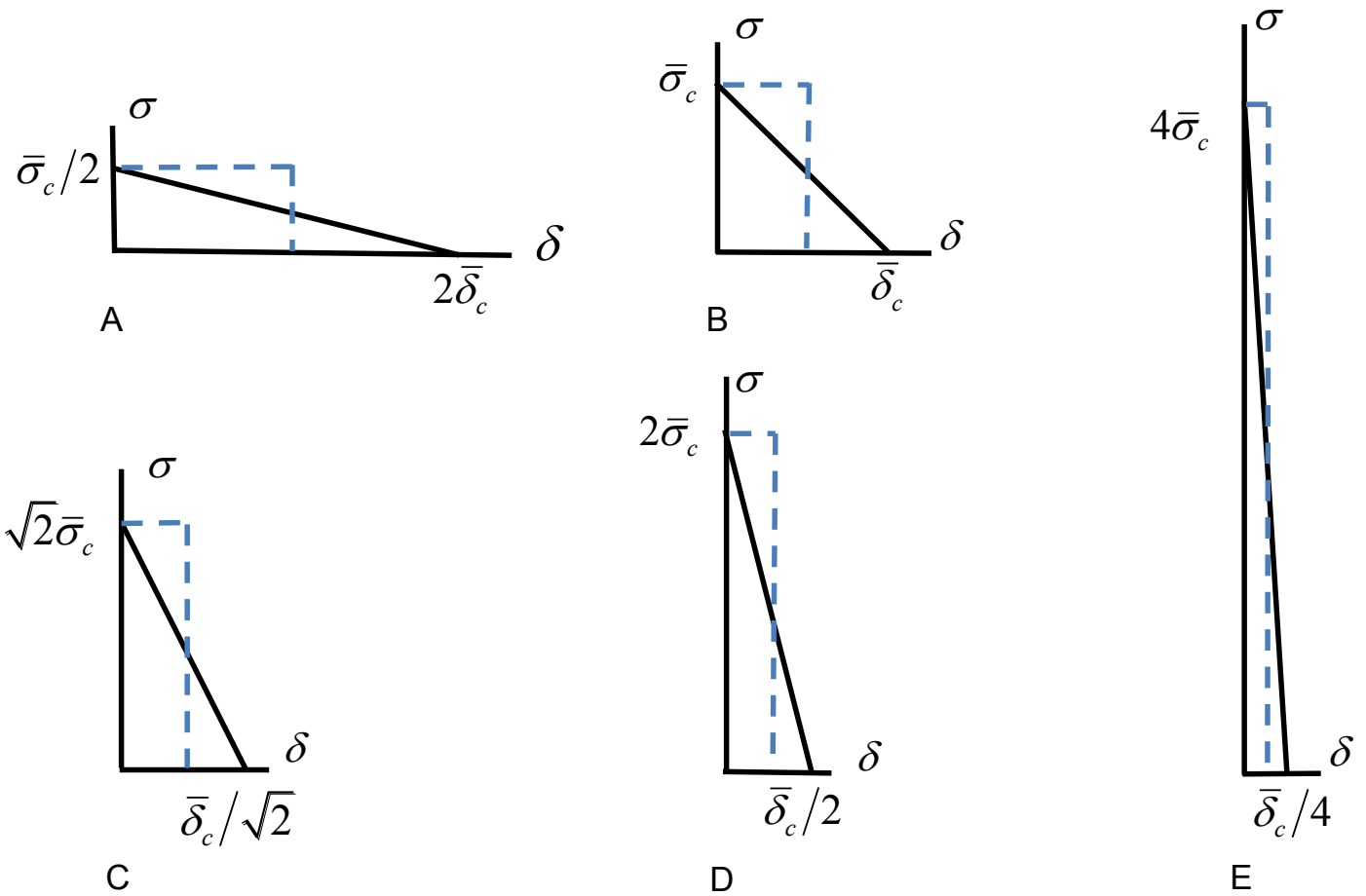

Linear Softening Model: Solid Line Dugdale Model: Dashed Line

Fig. 4 Five cohesive laws with different maximum tractions but the same cohesive work rate for both the Linear Softening and the Dugdale Models. 

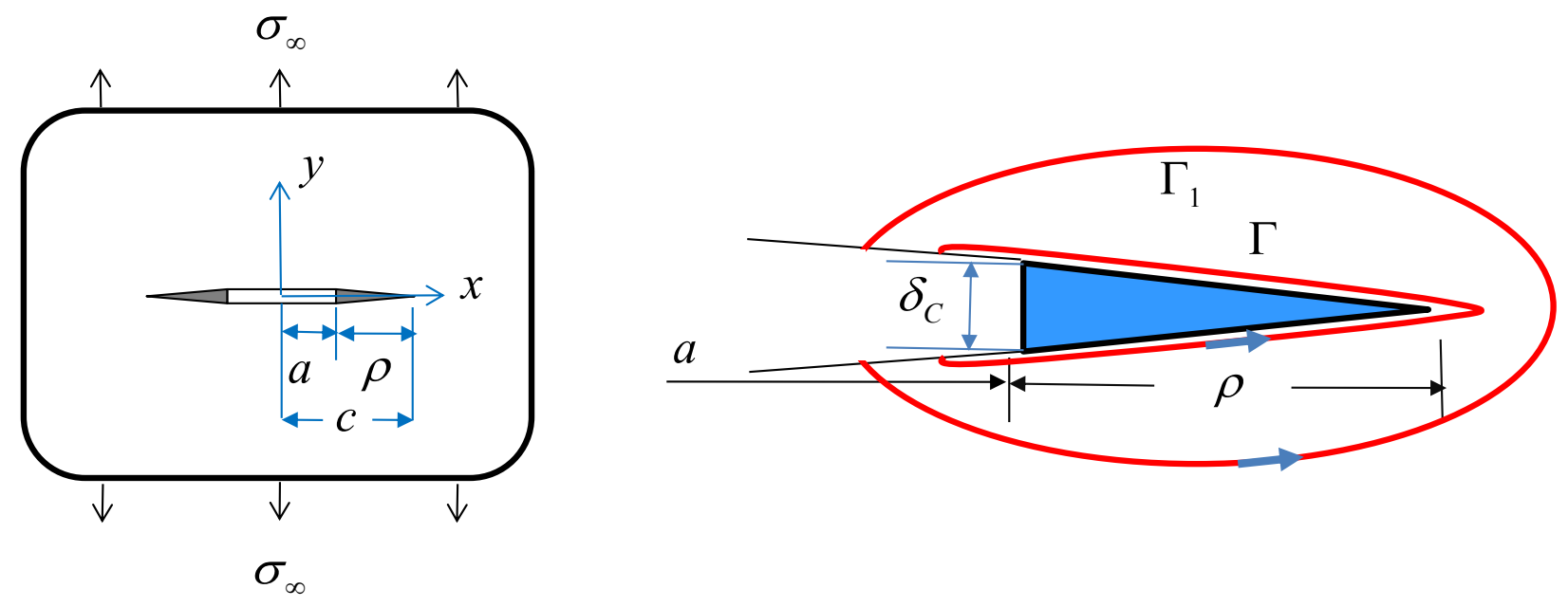

Fig. 5 J-integral paths around a cohesive zone.

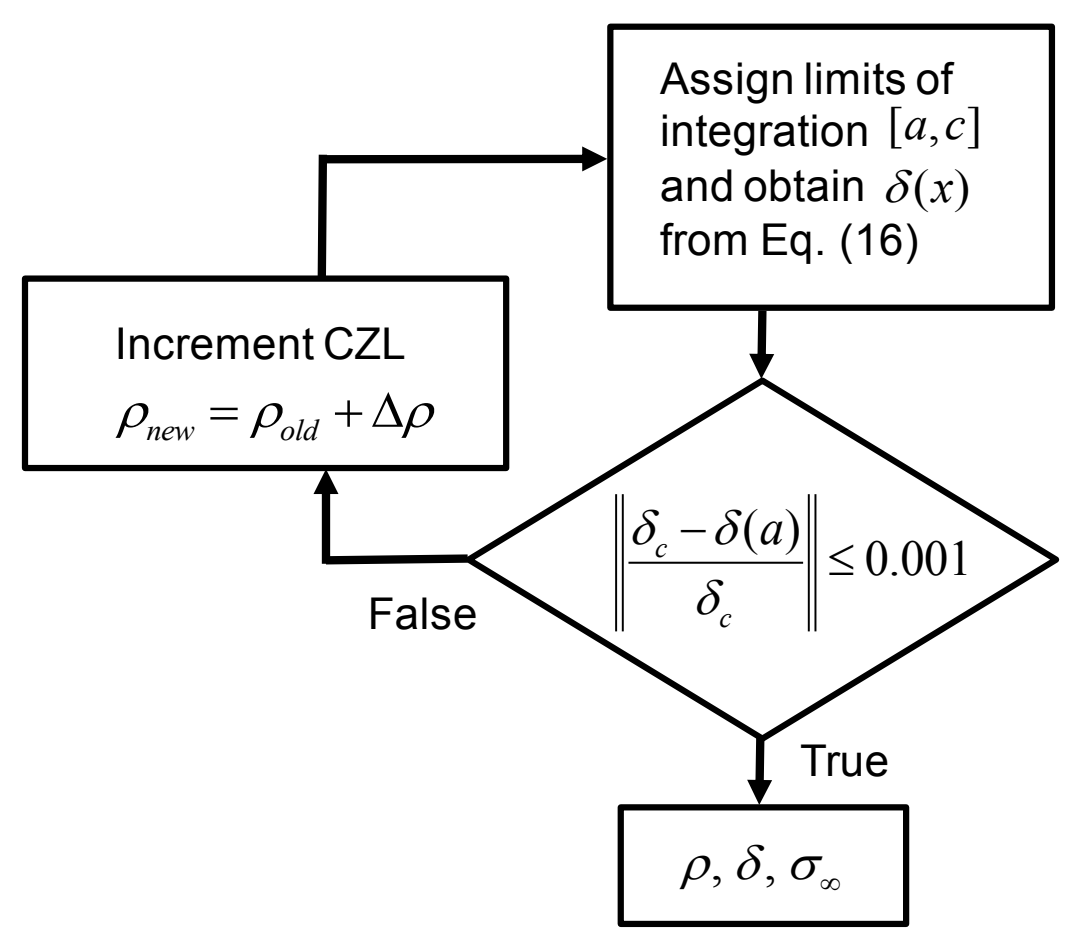

Fig. 6 Iterative solution procedure (CZL:cohesive zone length). 


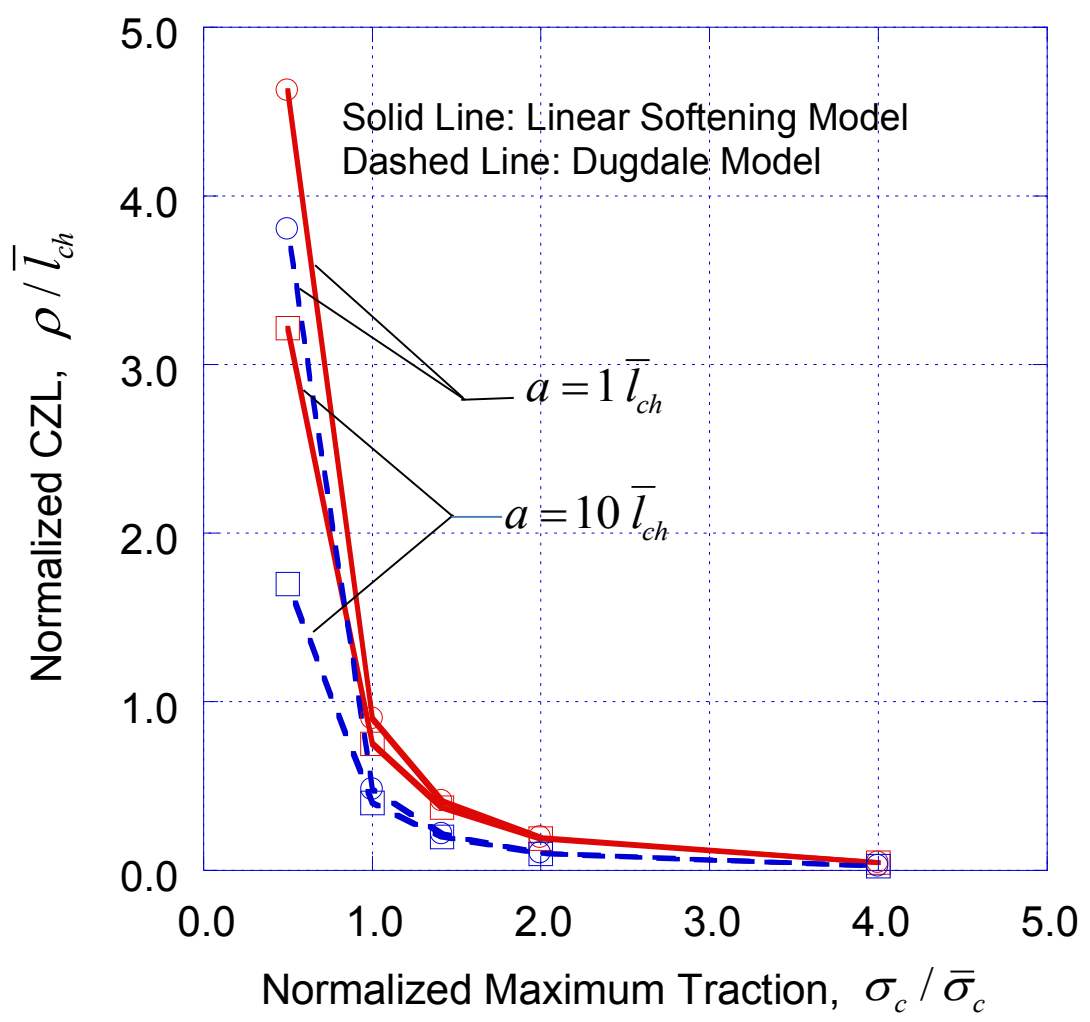

Fig. 7 Cohesive zone length as a function of maximum traction for two different crack lengths.

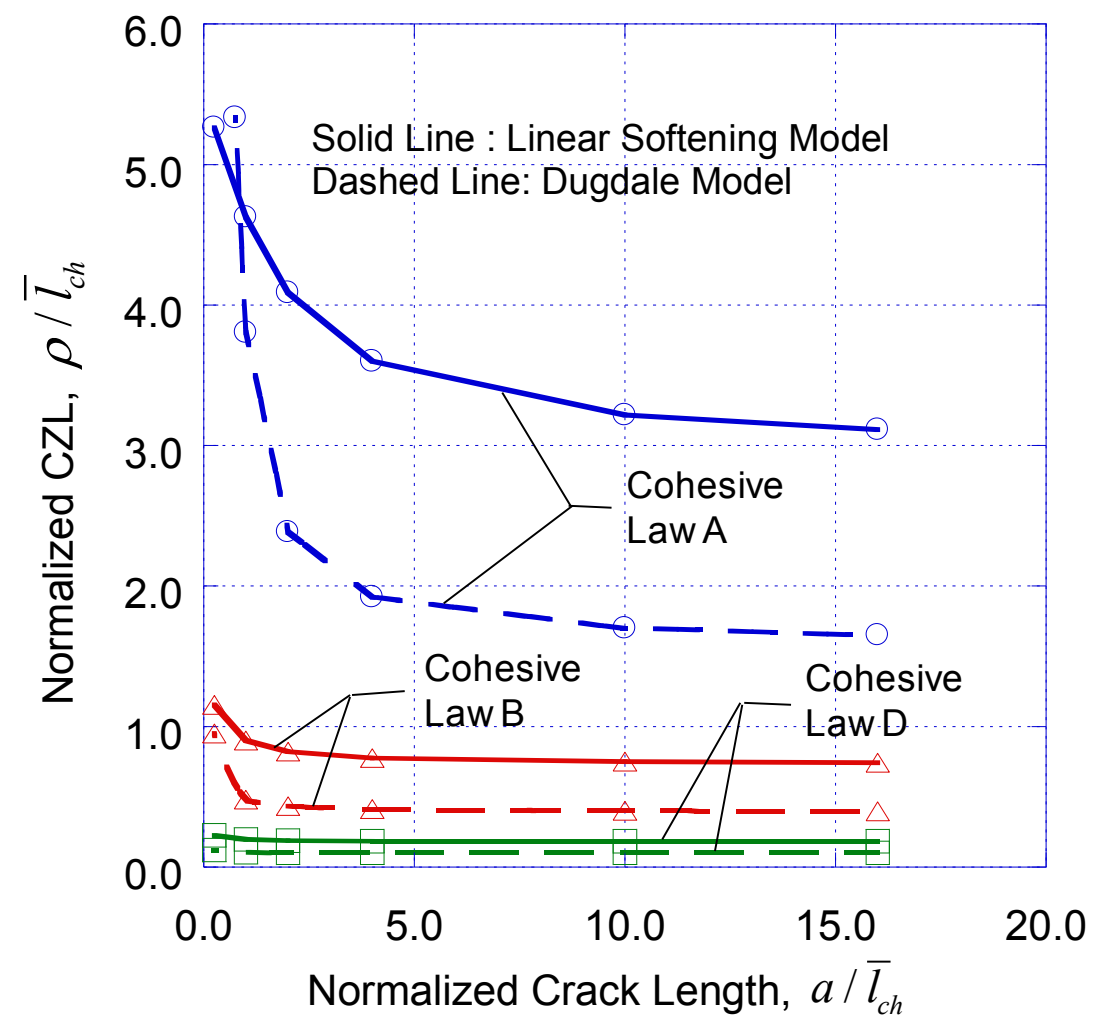

Fig. 8 Cohesive zone length as a function of crack length for cohesive laws with different maximum tractions. 


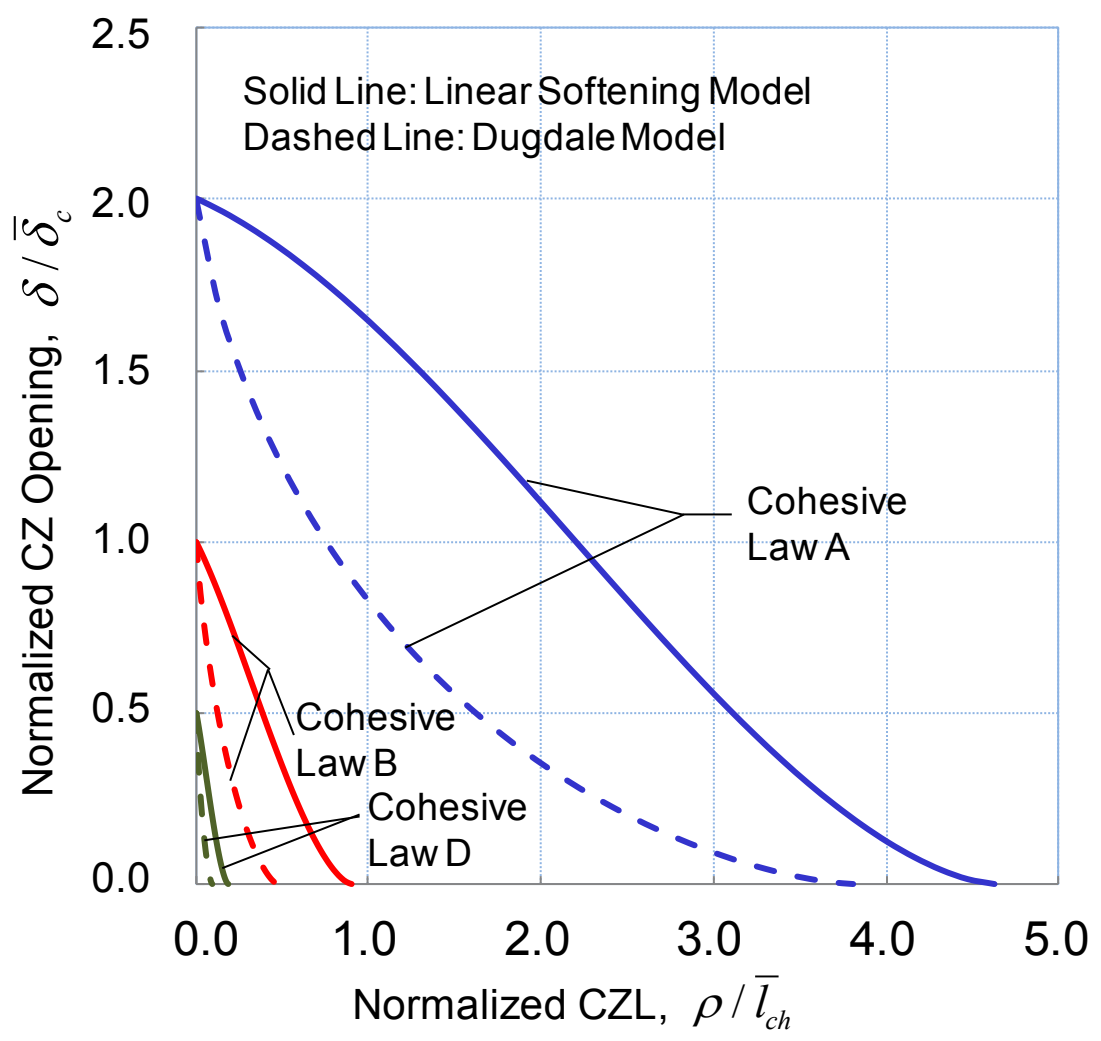

Fig. 9 Cohesive opening displacements along the cohesive zone length for cohesive laws with different maximum tractions $\left(a=1 \bar{l}_{c h}\right)$.

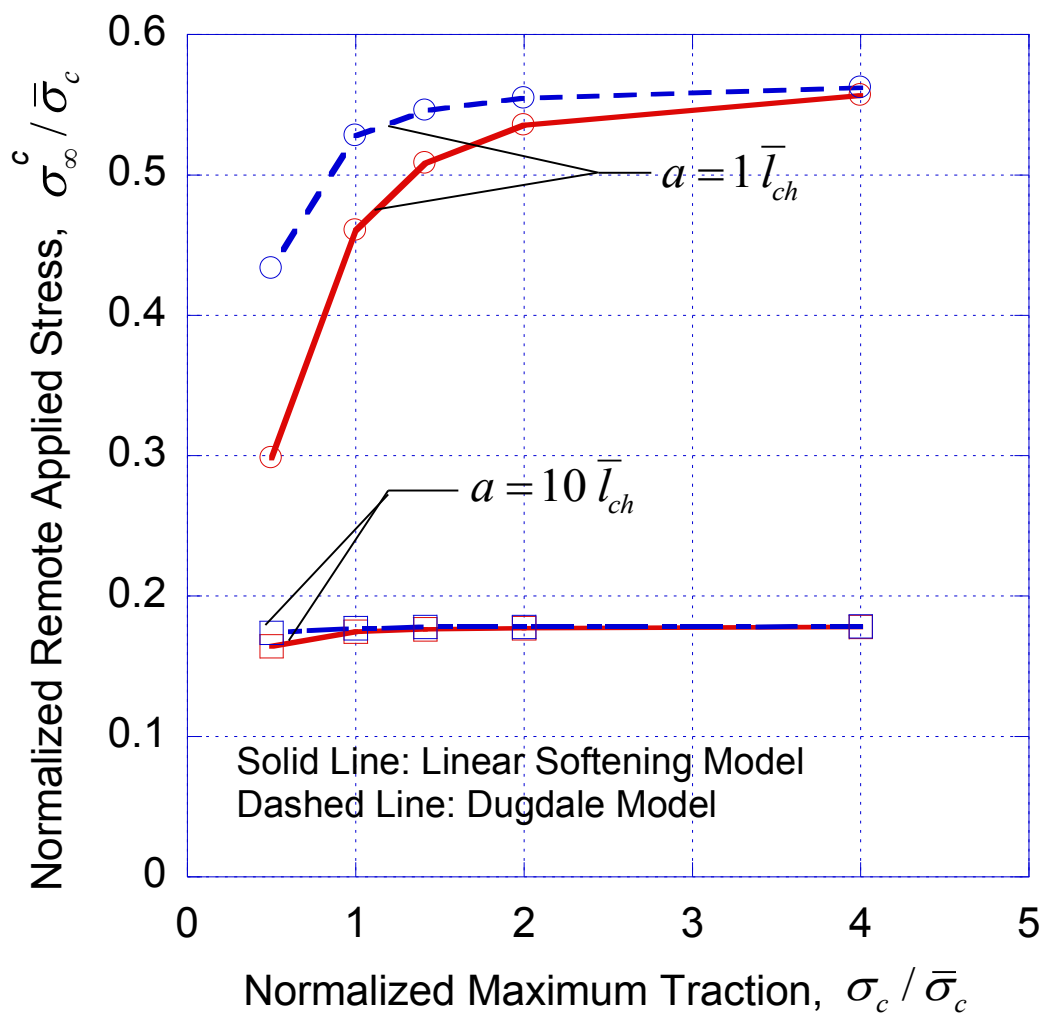

Fig. 10 Critical remote applied stress as a function of maximum traction for two different crack lengths. 


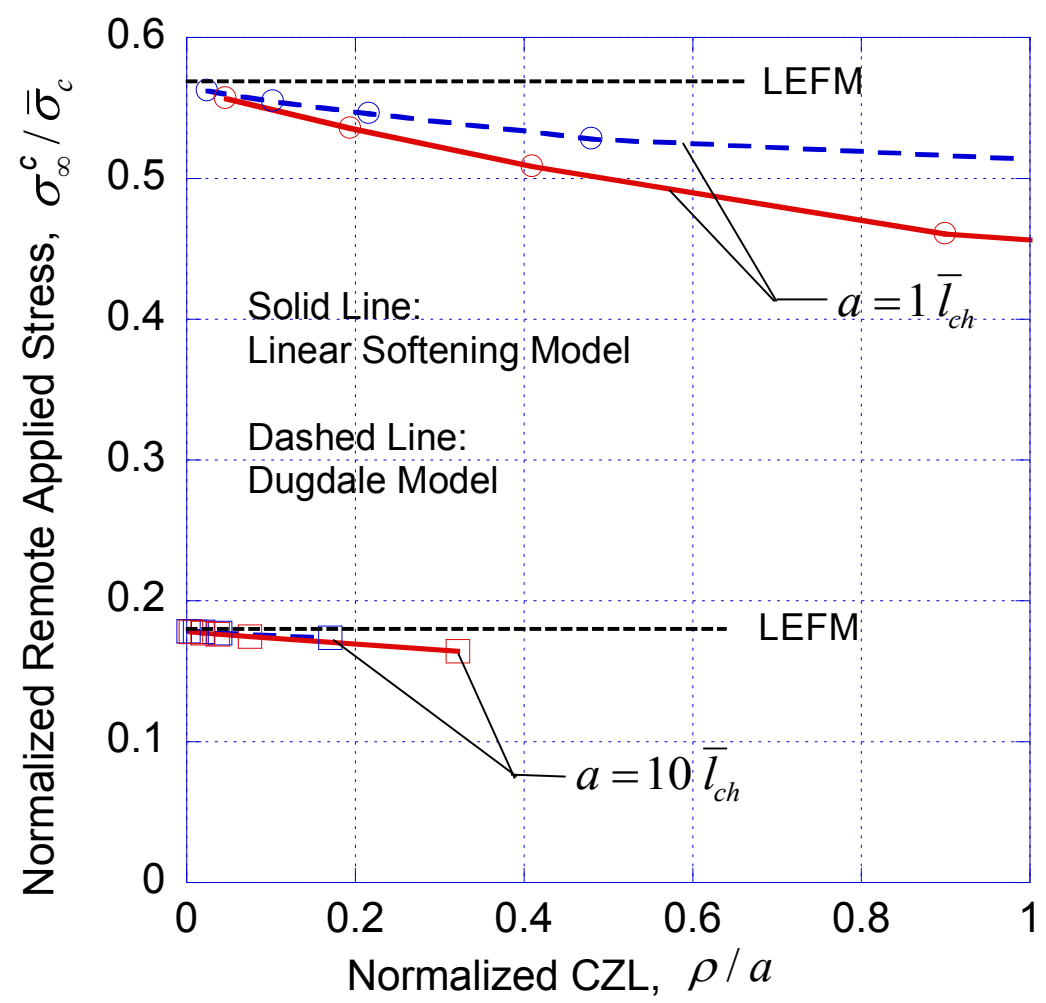

Fig. 11 Normalized critical remote applied stress as a function of the normalized cohesive zone length.

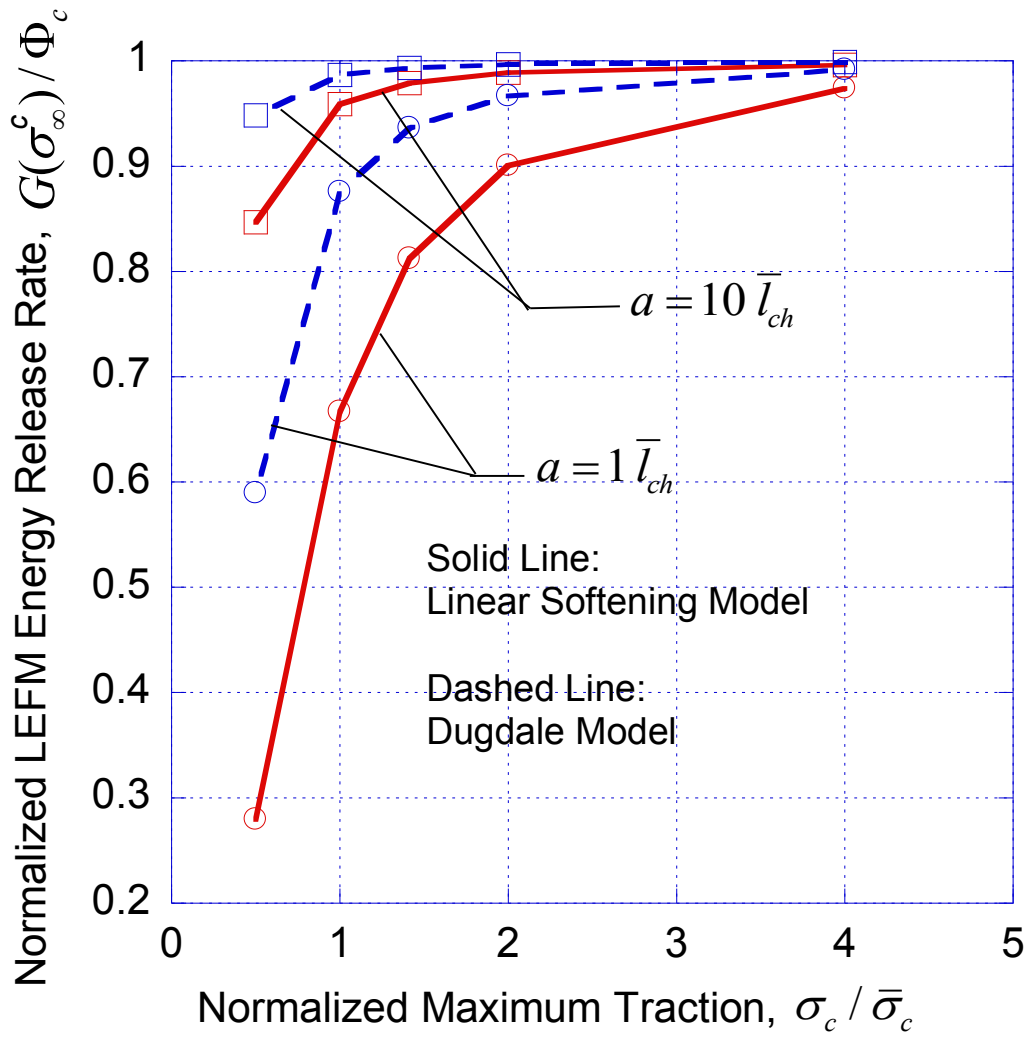

Fig. 12 Normalized LEFM energy release rate as a function of normalized maximum traction. 


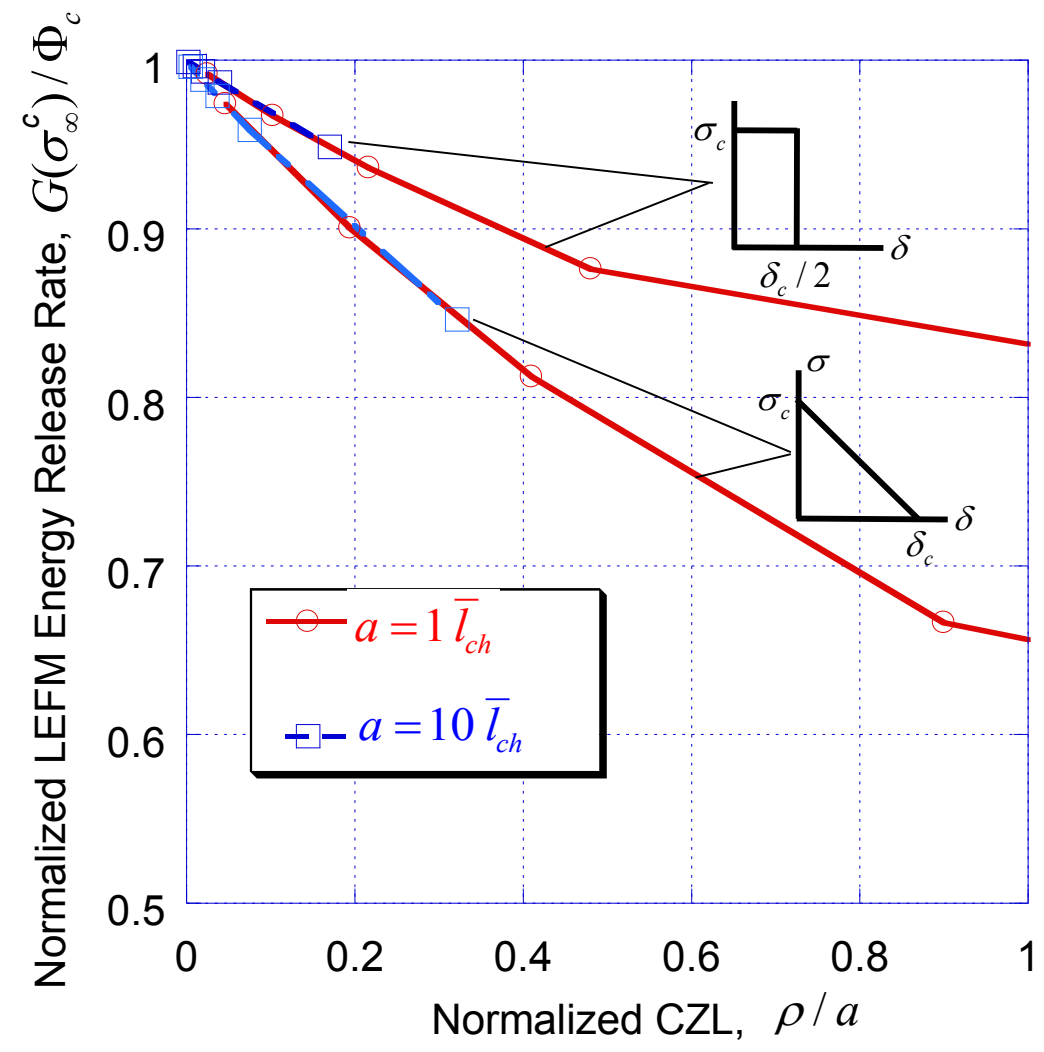

Fig. 13 Normalized critical LEFM energy release rate as a function of normalized cohesive zone length.
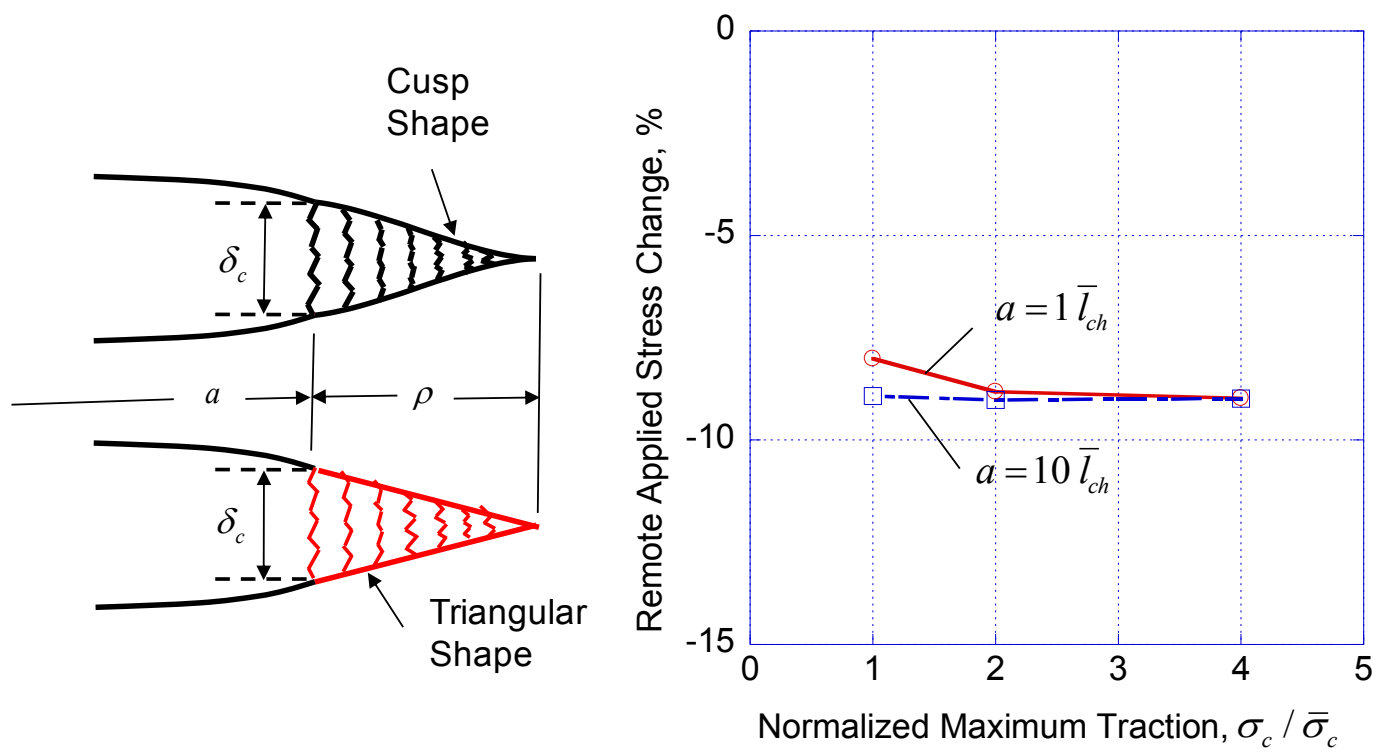

Fig. 14 Changes of remote applied stress due to modifying the cohesive zone opening profile from a cusp shape to a triangular shape. 